\title{
Fabrications and Structural Characterization of Ultra-fine Carbon Fibers by Electrospinning of Polymer Blends
}

\author{
C. Kim ${ }^{\mathrm{a}}$, Y. J. Cho ${ }^{\mathrm{a}}$, W. Y. Yun ${ }^{\mathrm{a}}$, B. T. N.Ngoc ${ }^{\mathrm{a}}$, K. S. Yang ${ }^{\mathrm{a}}$, D. R. Chang ${ }^{\mathrm{b}}$, J. W. Lee ${ }^{\mathrm{c}}$, M. \\ Kojima $^{\mathrm{d}}$, Y. A. Kim ${ }^{\mathrm{d}}$, M. Endo ${ }^{\mathrm{d}}$
}

\begin{abstract}
${ }^{a}$ Center for Functional Nano Fine Chemicals, Chonnam National University 300 Yong-bond dong, Buk-gu, Gwangju 500-757, Korea.

${ }^{\mathrm{b}}$ Korea Institute Industrial Technology (KITECH), Materials \& Components R\&D team, 97135 Wolchul-dong, Bukgu, Gwangju, 500-460, Korea

'Department of Chemical Engineering, Seonam University, Namwon 590-170, Korea

${ }^{\mathrm{d}}$ Faculty of Engineering, Shinshu University, 4-17-1 Wakasato, Nagano-shi 380-8553, Japan
\end{abstract}

\begin{abstract}
Here we demonstrated the ability to fabricate ultra-fine carbon fibers through electrospinnig of the polymer blends of the polyacrylonitrile (PAN) and polyamic acid (PAA) and the subsequnt thermal treatment. The good spinnability of the polymer blends was achieved by reducing the solution viscosity with the addition of PAN. There was a close correlation between the fiber diameter developed in the spinning process and the physical properties of the carbonized fibers; the smaller in fiber diameter gave rise to the higher crystallinity of the single carbonized fiber and, furthermore, the higher electrical conductivity and mechanical strength of the carbonized fiber web. High anisotropy in mechanical strength of the carbonized fiber webs was ascribed to the partial alignment of fibers along the winding direction in the electrospinning process.
\end{abstract}

Keywords: A. Carbon fibers, B. Carbonization, D. Electrical properties, Mechanical properties

*Corresponding author. Tel.: +81-26-269-5212; Fax: +81-26-269-5208,

E-mail: yak@endomoribu.shinshu-u.ac.jp (Y.-A. Kim) 
Carbon fibers with high specific strength, low weight and high transport properties have been utilized as a strunctural reinforcement in a wide range of fields from spaceshuttle, army to sports goods [1]. It is well known that the structure and properties of the carbon fibers are highly depending on the chemical nature of the precursors [2]. Thus, the judicious selection of the precursors in combind with the optimized fabrication processes (i.g., spinning, stabilization and carbonization) could be one of the way to get high performance carbon fibers. Unfortunatley, the current spinning system have their intrinsic limitation for preparing ultrathin carbon fibers with diameter below $1 \mu \mathrm{m}$, and also have problem for spinning polymer blends containing highy different molecular structures.

To solve these problmes, we have selected the recently highlighted electrospinning system because this technique has shown to be a simple but powerful technique for the preparation of fibers with sub-micrometer diameters [3, 4]. In addition, the electrospun fibers hold promise in various applications from tissue engineering, sensor, protective clothing to high-performance filter etc [5-8]. Up to now, there was a few reports on the synthesis of electrospun-derived carbon nanofibers from the neat polyacrylonitrile (PAN) as flexible polymer [9-15] and the neat polyamic acid (PAA) as rigid polymer [16, 17], respectively. Unfortunaltely, there were limited studies on the electrospinning of polymer blends [18, 19] even though blending technique enable us to tailor the diameter and also texture of the obtained fibrous carbons. In this study, we demonstrated the ability to fabricate ultra-fine carbon fibers from polymer blends (PAN/PAA) with highly different molecular structure via a electrospinning process and the subsequent thermal treatment at $1000^{\circ} \mathrm{C}$ in an inert atomosphere. This study reveals that the diameter and properties of the carbonized fibers are stronlgy depending on the blend ratio of two polymers.

PAN/PAA blend solutions were prepared by dissolution of pyromellitic dianhydride (PMDA) in previously dissolved in PAN in $N, N$-dimethylformamide (DMF) and followed by additional dissolution of benzidine in one to one molar ratio, and then stirred with mechanical stirrer until the reaction completed. The blend ratios of PAN/PAA were controlled through varying relative weight ratios of PAN/PAA as $10 / 0,7 / 3,5 / 5,3 / 7$, and $0 / 10$ to be total polymer concentration of 10 wt. \% in the DMF solvent. The blend solutions were spun into fiber webs through a positively charged capillary using an electrospinning apparatus at dc $20 \mathrm{kV}$ (NT-PS35K, NTSEE Co., Korea). The negative electrode was connected to a drum winder collecting the fiber web. The electrospun fibers were collected as web on an aluminum foil wrapped on the metal drum $($ diameter $=16 \mathrm{~cm}$ ) with a rotation speed of ca. $300 \mathrm{rpm}$. The webs were stabilized by heating the samples at a rate of $1{ }^{\circ} \mathrm{C} / \mathrm{min}$ up to $280^{\circ} \mathrm{C}$ and holding for 1 hour under air 
current. The stabilized fiber webs were carbonized at a rate of $5^{\circ} \mathrm{C} / \mathrm{min}$ up to $1000^{\circ} \mathrm{C}$ in a tubular electrical furnace under an argon atmosphere.

The fiber morphology and diameter distribution of the PAN/PAA polymer blends-derived electrospun fibers are studied using field emission-scanning electron microscope (FE-SEM) (note that count number is over 100). Pure PAN-derived electrospun ultra-thin fibers have small-sized and homogenous diameter distribution and long fibrous morphology (Fig. 1 (a)). On the other hand, pure PAA-derived fibers contain (Fig. 1 (e)) irregularly shaped and rather short fibrous morphology in a wide distribution of diameters ranged from few hundred nanometers to $3 \mu \mathrm{m}$, possibly due to their partial phase separation of the PAA solution. It should be note that we are able to obtain long and straight fibrous shape from PAN/PAA polymer blends. In addition, diameter distributions of electrospun ultra-thin fibers increase with an increase in concentration of PAA in polymer blends (see Fig. 1). In order to better understanding of the spinnability of blend polymers, the viscosities of the polymer solutions in DMF were measured by a cone and plate rheometer (DV II, Brookfield Co.) with spindle number of 3. All polymer solutions exhibit typical non-Newtonian behaviors that are shear-thinning with an increase in shear rate (Fig. 2). The viscosity value decrease as the concentration of the PAN increased. Therefore, it is certified that the reduced average diameter of the electrospun ultra-thin fibers is the result from the improved spinnability of polymer blends by reducing solution viscosity with the addition of the PAN polymer $[15,16]$.

From FT-IR (Nicolet 520p, USA) study on electrospun fibers, no new peak appearance indicates that only the physical blends occurs instead of chemical reaction between PAN and PAA in electrospun fibers. Thermal behaviours of electrospun ultra-thin fibers in nitrogen atmosphere were evaluated by thermo-gravimetric analysis (TGA, STA 1640, Stanton Redcroft). Pure PAA-derived fibers in a nitrogen atmosphere lose weight in three ranges (i.g., below 100, $100-200$ and $550-650^{\circ} \mathrm{C}$ ), which ranges are likely to indicate solvent evaporation, imidization or stabilization, and carbonization, respectively (Fig. 3). On the other hand, the weight changes in case of pure PAN-derived fibers occur largely around $300^{\circ} \mathrm{C}$, and stabilize for temperature region above $400^{\circ} \mathrm{C}$. The thermal behaviours of the blend polymer-derived fibers show a combination of pure PAN and PAA. Based on the FT-IR and TGA analysis, it is clear that the PAN/PAA blend fiber-derived electrospun ultra-thin fibers present the typical nature of PAN and aromatic PI materials. When heated in nitrogen to temperatures above $700^{\circ} \mathrm{C}$, the carbonization yield of PAN/PAA blend fibers show ca. $40 \%$, indicating a relatively high thermal stability. In addition, when they were exposed to steam at $800^{\circ} \mathrm{C}$ for 30 minutes, blend-derived carbon fibers 
exhibited the highest specific surface area of ca. $1046 \mathrm{~m}^{2} / \mathrm{g}$ (pure PAN $=700 \mathrm{~m}^{2} / \mathrm{g}$ ), possibly due to their heterogeneous textures.

For the carbonized electrospun ultra-thin fiber webs at $1000^{\circ} \mathrm{C}$, we carried out systematic analysis in terms of their microstructure and physical properties. Figure 4 show stress-strain curves for the carbonized electrospun fiber web in parallel to winding direction (Fig. 4 (a)) and to perpendicular direction (Fig. 4 (b))) by using micro-strain tester (MST-I, Shimazu). The tensile strength of the pure PAN- and PAN/PAA (=7/3)-derived carbon fiber webs is twice as high as that of pure PAA-derived carbon fibers, and in addition, no large changes in strain-atbreak (ca. 2.2) is observed. Possible explanations for this data are: (1) the decreased diameter of a single carbon fiber, and (2) in addition, increased fiber-to-fiber contact point in the carbonized fiber web (see average diameter of the carbonized nanofibers in Table 1). In addition, high anisotropy of the mechanical properties at two different directions is ascribed to partial alignment of carbon fiber along the winding direction.

In order to evaluate the structural changes by thermal treatment, we carried out Raman, XRD study and electrical conductivity measurement for the carbonized electrospun fiber webs. The data representing structural factors and physical properties are summarized in Table 1 . The electrical conductivity and crystallinity (higher (002) diffraction line and $R$ value) increase linearly with an increasing concentration of PAN. On the contrary, carbon yield of the PAN/PAA blend polymer-derived carbonized fiber increase with an increase in PAA concentration. The improved spinnability derived from the reduced viscosity of the polymer blend solution by adding PAN leads to the smaller fiber diameter, due to the stronger deformation during the electrospinning, which might give rise to the higher alignment of molecular chains along the fiber direction. From these experimental results, it is proved that the fiber diameter controlled by the electrospinning conditions strongly affects the physical and electrical properties of the carbonized ultra-thin carbon fibers. Therefore, the combination of the increased crystallinity of the singe carbon fiber and the increased fiber-to-fiber contact density of the carbonized fiber, intrinsically due to the small-sized fiber diameter, leads to higher tensile strength and higher electrical conductivity of the carbonized fiber web. Further studies have to be done for understanding the expected two-phased textures in a single carbon fiber because two polymeric components might experience highly different structural changes during air stabilization and as well carbonization.

In summary, we successfully fabricate ultra-thin carbon fiber webs by electrospinning PAN and PAA polymer blends and subsequent air-oxidation and thermal treatment processes, and 
then evaluate their structural and physical properties by using various analytic techniques. The ability to form straight and long fibers from the polymer blend is achieved by decreasing solution viscosity with the addition of PAN. This leads to the formation of the smaller fiber diameter. The smaller in fiber diameter indicates the higher stretching rate experienced during the electrospinning because it is well known that the electrically charged jet elongate in accordance with the evaporation of solvent until the attachment on the winding drum. More specifically, the smaller in the fiber diameter, the higher degree of molecular chain orientations along the fiber length within a single electrospun fiber is expected due to the stronger deformation during the whipping mode. The two structural factors (e.g., fiber diameter and chain orientation) developed in the spinning process are believe to determine the physical properties of the carbonized fibers. The expected higher degree of polymer chain orientation in smaller fiber diameter leads to the higher crystallinity (lower $\mathrm{R}$ value) of the carbonized fibers. Consequently, the smaller in fiber diameter and the higher crystallinity in a single fiber in combined with an increased fiber-to-fiber contact ratio in fiber web give rise to higher electrical conductivity and as well higher tensile strength of the carbonized fiber web.

\section{Acknowledgments}

This work was supported by the Next Korea Institute of Industrial Technology (KITECH). One of us (Lee) acknowledges the support by grant No R01-2005-000-10742-0 from the Korea Science \& Engineering Foundation. This work was supported by the CLUSTER of Ministry of Education, Culture, Sports, Science and Technology in Japan. 


\section{References}

[1] T. D. Burchell, Carbon Materials for Advanced Technologies, PERGAMON: New York, 1999.

[2] D. D. L. Chung, Carbon Fiber Composites, Butterworth-Heinemann, New York, 1994.

[3] A. Formhals, US Patent 1,975,504, 1934.

[4] D. Li, Y. Xia, Adv. Mater. 16 (2004) 1151.

[5] P. Gibson, H. S. Gibson, R. Rivin, Colloids Surf. A. 187-188 (2001) 469.

[6] D. Aussawasathien, J. H. Dong, L. Dai, Synth. Met. 154 (2005) 37.

[7] Y. H. Lee, J. H. Lee, I. G. An, C. Kim, D. S. Lee, Y. K. Lee, J. D. Nam, Biomaterials 26 (2005) 3165.

[8] P. P. Tsai, H. S. Gibson, P. Gibson, J. Electrostatics 54 (2002) 333.

[9] I. Chun, D. H. Reneker, Adv. Mater. 31 (1999) 36.

[10] Y. Wang, J. J. Santiago-Aviles, J. Mater. Sci. Lett. 21 (2002) 1055.

[11] C. Kim, K. S. Yang, Appl. Phys. Lett. 83 (2003) 1216.

[12] Y. Wang, J. J. Santiago-Aviles, R. Furlan, I. Ramos, IEEE Trans Nanotech 2 (2003) 39.

[13] Y. Wang, S. Serrano, J.J. Santiago-Aviles, Synth. Met. 38 (2003) 423.

[14] S. Y. Gu, J. Ren, Q. L. Wu, Synth. Met. 155 (2005) 157.

[15] S. Y. Gu, J. Ren, G. J. Vancso, European Polymer Journal 41 (2005) 2559.

[16] K. S. Yang, D. D. Edie, D. Y. Lim, Y. M. Kim, Y. O. Choi, Carbon 41 (2003) 2039.

[17] C. Kim, Y. J. Kim, Y. A. Kim, Solid State Communications 132 (2004) 567.

[18] E. Zussman, A. L. Yarin, A. V. Brazilevsky, R. Avrahami, M. Feldman, Adv. Mater. 18 (2006) 348.

[19] C. Kim, Y. I. Jeong, B. T. N. Ngoc, K. S. Yang, J. W. Lee, M. Kojima, Y. A. Kim, M. Endo, Small (2006) DOI: 10.1002. 


\section{Figure captions}

Figure 1 FE-SEM images and their corresponded diameter distributions of the pure PAN-, the pure PAA-, the PAN/PAA blend polymer-derived fiber webs at different blending ratios: (a) PAN/PAA=100/0, (b) PAN/PAA=70/30, (c) PAN/PAA=50/50, (d) PAN/PAA=30/70, (e) $\mathrm{PAN} / \mathrm{PAA}=0 / 100$. Arrows in all images indicate the winding direction of drum.

Figure 2 Variations of solution viscosities as a function of shear rate for three polymers blends of PAN/PAA= 70/30, 50/50 and 30/70, respectively.

Figure 3 Thermo-gravimetric analyses of the pure PAN-, the pure PAA-, PAN/PAA blend polymer-derived fiber webs in a nitrogen atmosphere.

Figure 4 Stress-strain curves of the carbonized electrospun fiber webs at different directions (a) parallel to the winding direction (b) vertical to the winding direction. 